\title{
Technologie(s) de la formation
}

\author{
Albero Brigitte \\ Université Européenne de Bretagne - Rennes 2 (CREAD, EA 3875)
}

In : Jorro A. (ed.) (2014). Dictionnaire des concepts de la professionnalisation. Bruxelles : De Boeck, pp. 309-313.

\section{1 - Présentation du concept}

Dans son sens courant en éducation et formation, le terme "technologies" (pl.) renvoie à l'ensemble des objets techniques, anciens ou récents, utilisés par les acteurs du domaine. Depuis les simples auxiliaires de l'action en tant qu'objets à fonction ancillaire, ce terme désigne avec la banalisation du numérique tout un champ d'expériences et de pratiques étroitement rattachées à la notion d'innovation. Devenus directement dépendants de produits industriels qui interviennent de façon toujours plus profonde dans le fonctionnement mental et les processus humains de connaissance, ces " objets " s'avèrent de plus à plus complexes à délimiter (à la fois matière, énergie, langage, symbole et réseau) et à maîtriser dans leurs conséquences sur la conduite humaine (modèles sous-jacents, matrices d'action, systèmes d'interrelations, jeux de dépendances, etc.), aussi bien que sur l'environnement naturel et social.

Au sens étymologique, le terme " technologie " (tecknè / logos) (sg) réfère à l'ensemble des théories et des discours, des modèles, enquêtes et analyses qui portent sur les techniques en tant que domaine d'étude. Ce terme renvoie ainsi au champ de recherche qui s'attache à décrire, comprendre et expliquer les relations entre les caractéristiques techniques des objets et les dimensions spécifiquement humaines de leur usage, en particulier en éducation et en formation.

Habituellement réduit à la discipline scolaire du même nom, étudiée en tant que telle par certains chercheurs (Lebeaume, 2008), ce champ de recherche est en réalité si riche et si diversifié qu'il est difficile de lui attribuer un périmètre lisible en sciences de l'éducation (SE) et plus largement en sciences humaines et sociales (SHS). Pour ne prendre que les SE, il est éclaté en de multiples secteurs structurés par des intérêts variés : supports (papier, analogique, numérique); média (radio, télévision, internet); objets matérialisés (multimédia, ordinateur, tableau interactif); terrains d'enquête (scolaire, supérieur, formation d'adultes); échelles d'analyse (micro, meso, macro); modalités de formation (présentiel, hybride, distance) ; rapports aux institutions (formel, non formel, informel); types de rationalisation (référentiels, grilles d'analyse, ingénieries diverses); types de centration (enseignement, formation, apprentissages).

A ce premier niveau de diversité s'ajoutent des divergences de fond quand il s'agit de positionner l'élaboration de connaissance : dans un paradigme théorique (béhavioriste, computationnel, connexionniste, structuraliste, socioconstructiviste, enactif) et méthodologique (expérimentaliste, comportementaliste, ethnographique, phénoménologique, clinique); en proximité avec une discipline universitaire autre que les SE (anthropologie, histoire, information et communication, philosophie, psychologie, sociologie) ; dans une option de la pluridiscipline (mono-, inter- ou transdisciplinaire); selon une finalité (conceptualisation, théorisation, modélisation, optimisation des pratiques, développements techniques). 
A l'intérieur de cette diversité, on peut toutefois dégager quelques grandes orientations : propositions conceptuelles à visées épistémologique et théorique ; analyses plus ou moins extensives d'usages sociaux (enquêtes internationales, nationales, comparatives, longitudinales) ou intensives d'usages locaux plus strictement pédagogiques (analyse d'expériences et de pratiques) ; approches globales de l'activité visant à étayer le développement d'environnements techniques pour l'apprentissage humain (EIAH) ou bien les processus même d'apprentissage, dans une perspective didactique et/ou ergonomique.

C'est ainsi que, malgré les avancées et le foisonnement des connaissances produites, la technologie de la formation en tant que champ de recherche n'a pas encore réussi à intégrer dans une perspective d'ensemble la diversité de ses études et de ses méthodes. Tel que le considéraient déjà en leur temps A. Leroi-Gourhan (1936) et A.G. Haudricourt (1964), elle n'est pas encore devenue ce que la linguistique est aux langues, ou encore la sociologie aux faits sociaux, l'anthropologie aux cultures ou la psychologie aux différences de construction de l'intelligence et de l'acte d'apprendre entre individus. Le domaine n'en offre pas moins une abondance de concepts, de résultats validés et de moyens efficaces de compréhension qui éclairent, sous de multiples facettes, les relations de plus en plus interdépendantes et complexes entre instruments techniques et activités humaines.

\section{2 - Débats autour du concept}

La tendance à confondre le sens étymologique du terme technologie (sg) en tant que discours méthodique à propos des techniques et son sens courant ( $\mathrm{pl}$.) en tant que champ de pratiques a longtemps persisté dans le domaine de la formation. Dès les années 70 pourtant, les débats critiques relevaient l'ambiguïté sémantique d'une expression telle que technologies éducatives qui semblait accorder aux objets techniques une nature intrinsèquement formatrice. La confusion a conduit à soupçonner le secteur de prosélytisme faisant des chercheurs des militants technophiles. Depuis, l'explicitation des cadres théoriques et des méthodes d'enquête s'est développée dans les études ainsi que le recours explicite aux disciplines des SHS susceptibles d'étayer les analyses et les propositions conceptuelles. Parmi les apports du domaine, certains sont particulièrement significatifs et pourraient constituer des bases de connaissances partagées selon une double perspective épistémique et pragmatique.

Dans les décennies 1970-90, des travaux en SE ont mis en évidence pour chaque support technique (papier, son, image, multimédia) et chaque média (radio, télévision, internet) des spécificités conduisant à reconnaître qu'un message efficace sur un support ou un média ne l'est pas nécessairement sur un autre et doit être profondément retravaillé pour retrouver une efficacité (Jacquinot, 1977, sur le rapport entre texte et image ; Compte, 1998, sur la rhétorique de l'écriture télévisuelle; Baron, Bruillard, 1996, sur les particularités de l'informatique, notamment dans le secteur scolaire). Les processus à l'œuvre ont été conceptualisés autour des termes de médiatisation (traduction d'un média à l'autre) et de scénarisation (organisation des éléments du message à visée d'efficacité), nécessitant régulièrement une médiation humaine (Linard, 1989).

Par ailleurs, la mise en évidence des différences entre approche techno- et anthropo-centrée (Rabardel, 1995) a permis de comprendre que le point de vue adopté sur la relation entre objet technique et activité humaine avait une incidence sur la place relative de l'un et l'autre et l'efficacité cognitive de leur interaction. L'approche anthropocentrée qui accorde une grande importance à I'usage (Perriault, 1989) ainsi qu'au sens que le sujet donne à son activité (Linard, 1989), s'avère particulièrement pertinente en sciences de l'éducation. Elle permet d'appréhender les différences 
entre les processus de médiatisation (traduction d'un message sur différents supports selon leurs spécificités sémiotiques) et ceux de médiation (facilitation de l'accès au sens en vue de son appropriation par un humain) (Linard, ibid.). Elle permet aussi de distinguer les fonctions d'un artefact (objet fabriqué par le concepteur en vue d'une application pratique en fonction de ses schèmes de conception) et celles d'un instrument (objet mis en œuvre par l'utilisateur selon ses propres schèmes d'usage). En passant d'une analyse de l'action centrée sur l'objet technique à celle des relations que le sujet entretient avec celui-ci, l'approche anthropocentrée éclaire la différence entre les processus de l'instrumentation (lors de son utilisation, le sujet exploite et finalise les potentiels présents dans la structure de l'objet) et ceux de l'instrumentalisation (le sujet par son utilisation enrichit et dépasse les stricts potentiels de l'objet) (Rabardel, 1995).

Dans les décennies 1990-2010, la généralisation des objets techniques dans tous les domaines de la vie humaine rend banale leur présence dans les situations de formation, au point qu'ils finissent par ne plus être interrogés ni observés en tant que tels. Cette situation est nouvelle dans la socio-histoire du domaine. Si elle marque une avancée, elle représente également un risque. L'intégration de l'objet technique dans une relation de couplage avec l'activité est une avancée car elle conduit à une évolution des paradigmes d'analyse vers des approches plus trandisciplinaires: une approche que I'on pourrait qualifier en français de " médiationnelle " (Engeström, 2008) à partir des travaux de la psychologie historico-culturelle russe ; " enactive » (Durand, 2008) inspirée de la biologie ; ou encore " sociotechnique " (Albero, 2010) à la suite des travaux de l'anthropologie et de la sociologie des techniques. Le risque comporte deux versants. Il consiste, soit à minorer la fonction des objets dans la conduite des activités en accordant au sujet une autonomie d'intention et d'orientation de l'action qui est, en réalité, largement influencée par la structure même de l'objet, soit à minorer la part humaine de l'activité dans ses caractéristiques propres (chronobiologiques, socio-relationnelles ou émotionnelles par exemple) en les réifiant dans des systèmes d'interaction prédéterminés. Dans le premier cas, les analyses se centrent sur l'activité du sujet, l'objet devient transparent dans sa structuration, son langage propre et ses potentiels, il est traité comme un auxiliaire neutre de l'intention humaine, aucun des concepts d'analyse n'y fait référence et les caractéristiques de l'action finale sont imputées aux seules caractéristiques de la situation et des acteurs locaux. En privilégiant le couplage objet/activité, le second cas recourt à des concepts tels que "instance ", " entité " ou " actant " et tend ainsi à réifier les caractéristiques de l'action humaine, en minorant ses dimensions les plus spécifiques (créativité, détournement, interrogation, négociation, etc.) et en majorant l'analyse de logiques déterministes liées aux caractéristiques de l'objet (langages, modèles sous-jacents d'action, systèmes de contraintes, etc.).

Avec le développement continûment accéléré du numérique, la constitution d'une approche transdisciplinaire centrée sur l'analyse des causes et des conséquences de l'interdépendance toujours plus étroite entre techniques et humains s'avère indispensable. Pour le moins, pourrait-on imaginer que des rapprochements s'opèrent entre grands champs d'activité et de recherche (éducation et formation, information et communication, santé et soin, sports et loisirs, travail et professionnalisation, etc.), afin de dégager, à un méta-niveau d'analyse, des invariants, des grandes tendances et des moyens indispensables de compréhension pour piloter les problèmes soulevés par la puissance et la complexité des évolutions actuelles.

L'actualité récente dans divers domaines économiques et sociaux a montré qu'à défaut de cette connaissance, la logique instrumentale prévaut sur deux autres constitutives de l'action humaine : la logique axiologique en tant que sens et valeurs de l'action dans les projets collectifs et individuels ; la 
logique existentielle, en tant que sens et qualité de l'action pour le sujet selon la place qu'il occupe dans le groupe social. La question aujourd'hui est de savoir de quelle manière une approche à orientation scientifique peut contribuer à rendre intelligibles les environnements, les conditions et les processus par lesquels ces trois logiques peuvent fonctionner ensemble. La technologie dans le domaine de la formation se constituerait alors, selon les vœux d'A.G. Haudricourt (1964), en une science vraiment humaine...

\section{Bibliographie}

Albero, B. (2010). Penser le rapport entre formation et objets techniques : repères conceptuels et épistémologiques. Dans G. Leclercq, R. Varga, Dispositifs de formation et environnements numériques : enjeux pédagogiques et contraintes informatiques. Paris : Hermès/Lavoisier, coll. IC2, 37-69.

Baron G-L., Bruillard E. (1996). L'informatique et ses usagers dans l'éducation. Paris: PUF, coll. L'éducateur.

Compte C. (1998). La rhétorique télévisuelle : une médiation pour l'apprentissage des langues. Les Cahiers du CIRCAV (Centre Interdisciplinaire de Recherche sur les Communications Audiovisuelles), 10, 227-249.

Durand, M. (2008). Un programme de recherche technologique en formation des adultes. Une approche enactive de l'activité humaine et l'accompagnement de son apprentissage / développement. Education \& Didactique, 2 / 3, 97-121.

Engeström, Y. (2008). From teams to knots: Activity-theoretical studies of collaboration and learning at work. Cambridge: Cambridge University Press.

Haudricourt A-G. (1964). La technologie, science humaine. La Pensée, 115, 28-35.

Leroi-Gourhan A. (1936). L’homme et la nature. Encyclopédie française (T. VII, Section A, chap. 1). Paris : Larousse.

Jacquinot G. (1977). Image et pédagogie : analyse sémiologique du film à intention didactique. Paris : PUF, coll. L'éducateur.

Lebeaume, J. (2008). Les sciences à l'école : des leçons de choses à la technologie. Paris : Delagrave. Linard M. (1989, 1996, rééd. augmentée). Des machines et des hommes. Apprendre avec les nouvelles technologies. Paris : L'Harmattan, coll. Savoir et formation.

Perriault J. (1989). La logique de I'usage. Essai sur les machines à communiquer. Paris : Flammarion. Rabardel P. (1995). Les hommes et les technologies. Approche cognitive des instruments contemporains. Paris : Armand Colin. 